\title{
Clinical Importance of Carbapenemase Production in Gram-Negative Bacteria
}

Mandeep Kaur, Satish Gupte* and Tanveer Kaur

Department of Microbiology, Gian Sagar Medical College and Hospital, Rajpura, Punjab, India

\begin{abstract}
Carbapenem resistance is one of the major threats faced in antimicrobial treatment of infections caused by Gram negative organisms. In India and throughout the world the use of carbapenems has increased resulting in an increased resistance of pathogens to this class of antibiotics. Bacteria are capable enough to become resistant to antibiotics by a number of mechanisms both intrinsic and acquired, most common of which include enzymatic degradation of antibiotics. Carbapenem resistant Gram-negative bacteria usually spreads in the hospital settings to other patients and caregivers or relatives by unwashed hands or from contact with soiled equipment and surfaces such as bedrails, tables, chairs, countertops and door handles. These carriers are the ultimate sources of dissemination in the community. Detection of carbapenemase is a crucial infection control issue because they are often associated with extensive antibiotic resistance, treatment failures and infection-associated mortality. For the identification of carbapenemase producers different nonmolecular methods are used such as E- test (Epsilometer Test), Modified hodge test, MIC by Agar Dilution Method, Carba NP Test, EDTA disk synergy test, Boronic Acid Test, 2-mercaptopropionic acid inhibition (2-MPA) test. Molecular techniques remain the gold standard for the precise identification of carbapenemase genes. Most of these techniques are based on PCR and may be followed by a sequencing step if a precise identification of the carbapenemase gene is needed. This review article describes clinical importance and methods used for the identification of carbapenemase producers.
\end{abstract}

Keywords: Carbapenemases; Gram negative bacteria; PCR; E-test; Carba NP test

\section{Introduction}

Carbapenems (Imipenem, Meropenem, Ertapenem and Doripenem) have traditionally been reserved for therapy of suspected or confirmed infections with multi-drug resistant Gram negative bacilli, including extended spectrum $\beta$-Lactamase (ESBL) producing Enterobacteriaceae. In India and throughout the world the use of carbapenems has increased resulting in an increased resistance of pathogens to this class of antibiotics [1]. Bacteria are capable enough to become resistant to antibiotics by a number of mechanisms both intrinsic and acquired, most common of which include enzymatic degradation of antibiotics [2]. Carbapenemases are diverse enzymes that vary in their ability to hydrolyze carbapenems and other betalactams. Detection of carbapenemase is a crucial infection control issue because they are often associated with extensive antibiotic resistance, treatment failures and infection-associated mortality. Among the beta-lactamases, the carbapenemases, especially transferrable metallobeta-lactamases (MBLs) are the most feared because of their ability to hydrolyze virtually all drugs in that class, including the carbapenems.

The major concern is with transmissible carbapenemases. The transmissible enzymes can be acquired unpredictably by important nosocomial pathogens such as Pseudomonas aeruginosa, Acinetobacter baumannii and members of the family Enterobacteriaceae. The chromosomal enzymes occur predictably in less common pathogens such as Stenotrophomonas maltophilia, Aeromonas species, Chryseobacterium species and others [3]. The carbapenemase producing Gram negative bacteria spread easily between humans and acquire genetic material through horizontal gene transfer, mediated mostly by plasmids and by transposons [4]. Increasing resistance to carbapenems is now frequently being observed in many hospital acquired and several community-acquired infections [5].

\section{Clinical Importance}

The current worldwide emergence of carbapenem resistant Gramnegative bacteria constitutes an important growing public health threat. Although the presence of carbapenem resistant Gram-negative bacteria do not lead to the development of specific types of clinical infections, their importance to the clinician and the microbiologist are many. Most patients who are colonized with these organisms remain asymptomatic but they can serve as potential sources of infections following translocation across the intestinal lining or as a consequence of fecal contamination of wounds or devices [6]. To the microbiologist, presence of carbapenem resistant Gram-negative bacteria is important due to epidemiological concerns and concerns of resistance transfer in hospital and community settings. The presence of resistant bacteria in human intestines not only leads to sharing of resistance genes among themselves but can also acquire from or donate resistance genes to bacteria that are just passing through the intestine [7]. Carbapenemase producers are also multidrug resistant explaining the difficulty to treat infections. The heightened concern of carbapenem resistant Gramnegative bacteria is due to the understanding of the mechanisms of its spread and their implications in hospital and community based infections. Carbapenem resistant Gram-negative bacteria usually spreads in the hospital settings to other patients and caregivers or relatives by unwashed hands or from contact with soiled equipment and surfaces such as bedrails, tables, chairs, countertops and door handles. These carriers are the ultimate sources of dissemination in the community [8]. The outbreak of carbapenem resistant Gram-negative

*Corresponding author: Satish Gupte, Gian Sagar Medical College and Hospital Rajpura, Punjab, India, Tel: +9915025865; E-mail: drsatishgupte@hotmail.com

Received May18, 2015; Accepted June 11, 2015; Published June 18, 2015

Citation: Kaur M, Gupte S, Kaur T (2015) Clinical Importance of Carbapenemase Production in Gram-Negative Bacteria. J Trop Dis 3: 164. doi:10.4172/2329891X.1000164

Copyright: (c) 2015 Kaur M, et al. This is an open-access article distributed under the terms of the Creative Commons Attribution License, which permits unrestricted use, distribution, and reproduction in any medium, provided the original author and source are credited. 
bacteria in the hospital or the community, health care associated infections will not only become difficult to treat but will also escalate health care costs. Also community acquired infections previously treated easily such as pyelonephritis; appendicitis may become difficult to treat [8]. The prevention of spread of carbapenemase producers relies on early detection of carriers. CDC has recommended certain core measures for all acute and long-term care facilities with risk of carbapenem resistant Gram-negative bacteria transmission in hospital setting. Although general measures like hand hygiene, contact precautions, education of caregivers, isolation of known carbapenem resistant Gram-negative bacteria carriers and epidemiologic screening are vital to prevention of spread, active surveillance for the prevalence of carriage and transmission in the hospital setting is also an integral part of such a program [8].

\section{Carbapenemase Producing Gram Negative Bacteria in Correlation with Community}

Gram negative bacteria are normal intestinal flora and are important cause of most common clinical infections such as urinary tract infection, septicemia, pneumonia, meningitis, peritonitis, and device associated infections. They are often involved in community-acquired infections and are frequently observed to be multi-drug resistant. They have the propensity to spread easily between humans (hand carriage, contaminated food and water) and to acquire genetic material through horizontal gene transfer, mediated mostly by plasmids and transposons [9]. Enterobacteriaceae are common cause of community infections, raising the risk to spread of Carbapenem-resistant Enterobacteriaceae (CRE) into the community. These problems, combined with the limited treatment options have made CRE of public health important [10] Since 2000, spread of community-acquired enterobacterial isolates (Escherichia coli) that produce extended-spectrum $\beta$-lactamases (ESBLs) capable of hydrolyzing almost all cephalosporins except carbapenems has been reported worldwide [11]. The example of the spread of ESBL producers in the community within the past 10 years shows us that a high rate of carbapenemase producers in E. coli may be reached rapidly worldwide. Such community-based outbreaks will be difficult to control. Hand carriage is probably the biggest factor in transmission of extended spectrum $\beta$-lactamase producers, and there is little evidence to suggest that carriers of carbapenemase-producing K. pneumoniae would be different. Environmental contamination plays a limited role in transmission of the organism [12]. Caregivers should be aware that multidrug-resistant organisms of nosocomial origin can be transmitted in the community [13]. Modulation of the factors that enhance spread of carbapenemase producers in the community is difficult because these factors are multiple and are associated with lack of hygiene, overuse and over-the-counter use of antibacterial drugs, and increased worldwide travel. In addition, many carbapenemase producers carry unrelated drug-resistance determinants. So there is a need of rapid detection of Carbapenemase producing Gram negative bacteria in carriers to stop the risk of its spread.

\section{Detection of Carbapenemase Production in Gram- negative Bacteria by Different Methods}

\section{Non-molecular methods}

E test (Epsilometer Test): The E test is basically an agar diffusion method. The E test system comprises a predefined and continuous concentration gradient of different antimicrobial agents, which when applied to inoculated agar plates and incubated, create ellipses of microbial inhibition [14]. The E test utilizes a rectangular strip that has been impregnated with the drug to be studied. A lawn of bacteria is spread and grown on an agar plate and the E test strip is laid on top, the drug diffuses out into the agar, producing an exponential gradient of the drug to be tested. There is an exponential scale printed on the strip. After 24 hrs of incubation, an elliptical zone of inhibition produced and the point at which the ellipse meets the strip that is considered a reading for minimum inhibitory concentration (MIC) of the drug [15] (Figure $1)$.

MIC by agar dilution method: Agar dilution method used to determine the minimum inhibitory concentration of antimicrobial substances. Minimum inhibitory concentration is the lowest concentration of an antimicrobial that will inhibit the visible growth of a microorganism after overnight incubation. Minimum inhibitory concentrations are important in diagnostic laboratories to confirm resistance of microorganisms to an antimicrobial agent and also to monitor the activity of new antimicrobial agents [14]. A minimum inhibitory concentration is generally regarded as the most basic laboratory measurement of the activity of an antimicrobial agent against an organism [16]. Clinically, the minimum inhibitory concentrations are used not only to determine the amount of antibiotic that the patient will receive but also the type of antibiotic used, which in turn lowers the opportunity for microbial resistance to specific antimicrobial agents. Applying MIC testing to a number of bacterial strains in the same species provides an estimate of the concentration that inhibits $50 \%$ (MIC) and 90\% (MIC) of bacterial isolates and can indicate shifts in the susceptibility of bacterial populations to antibiotics [17]. In agar dilution method a varying concentrations of the antimicrobial agent are incorporated in series of agar plates onto which a standardized suspension of the test organism is inoculated. The drug dilution should be 10 times the required concentration. Mix $2 \mathrm{~mL}$ of each dilution with $18 \mathrm{ml}$ of molten agar at $50^{\circ} \mathrm{C}$ per plate. After incubation, the lowest concentration of the agent which shows no growth of test organism is the MIC.

Modified Hodge Test (MHT): Carbapenemase production is detected by MHT when the test isolate produces the enzyme and allows growth of a carbapenem susceptible strain (E. coli ATCC 25922) towards a carbapenem disk using Imipenem/Meropenem/Ertapenem

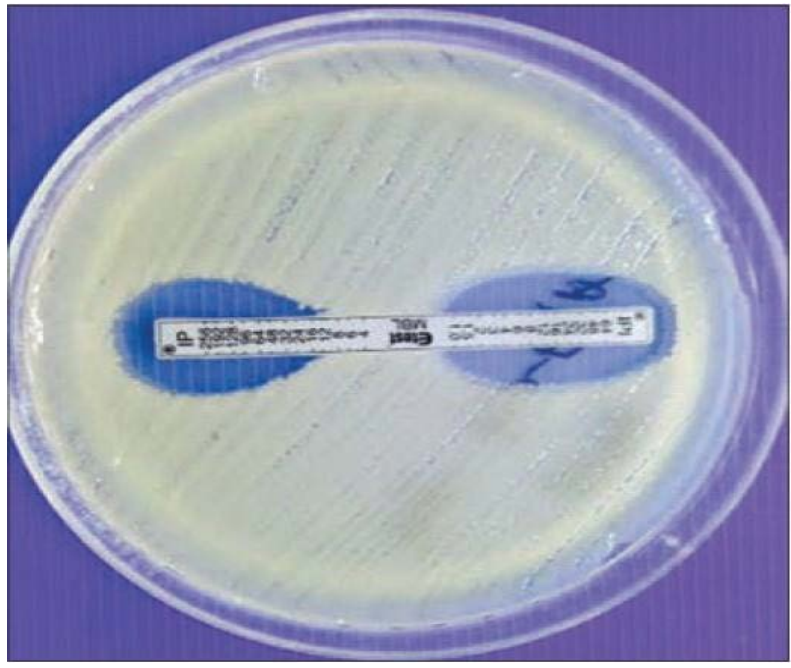

Figure 1: E-test showing carbapenemase production 
$(10 \mu \mathrm{g})$. The result is a characteristic cloverleaf like indentation [18]. Briefly, the growt is suspended in normal saline and matched to Mcfarland standard (0.5). This is diluted $1: 10$ by adding $0.5 \mathrm{ml}$ of the Mcfarland to $4.5 \mathrm{ml}$ of saline. E. coli ATCC 25922 is first inoculated on the Mueller Hinton Agar plate as lawn culture. Thereafter, carbapenem disc is placed in the centre. From the disc four different isolates (one known susceptible standard strain and one known resistant strain and two test strains) are streaked in a straight line radially. After incubation for $24 \mathrm{hrs}$ at $37^{\circ} \mathrm{C}$ in ambient air, the plates is examined for a clover leaftype indentation at the intersection of the test organism and the E. coli 25922, within the zone of inhibition of the carbapenem susceptibility disk. For quality control MHT Positive Klebsiella pneumoniae ATCC BAA-1708 and MHT Negative Klebsiella pneumoniae ATCC BBA 1706 are inoculated as radial streaks [19] (Figure 2).

Carba NP Test: Carba NP test is a rapid, sensitive and specific test for the identification of Carbapenemase-producing Gram-negative bacteria. The CarbaNP (Carbapenemase Nordmann-Poirel) test is performed as follows. One calibrated loop $(10 \mu \mathrm{L})$ of the tested strain directly recovered from the antibiogram is resuspended in a Tris- $\mathrm{HCl}$ $20 \mathrm{mmol} / \mathrm{L}$ lysis buffer, vortexed for 1 minute and further incubated at room temperature for 30 minutes. This bacterial suspension is centrifuged at $10,000 \mathrm{~g}$ at room temperature for 5 minutes. $30 \mu \mathrm{L}$ of the supernatant, corresponding to the enzymatic bacterial suspension, is mixed in a 96-well tray with $100 \mu \mathrm{l}$ of a $1-\mathrm{mL}$ solution made of 3 $\mathrm{mg}$ of imipenem monohydrate, $\mathrm{pH} 7.8$, phenol red solution, and 0.1 $\mathrm{mmol} / \mathrm{L} \mathrm{ZnSO}_{4}$. The phenol red solution is prepared by mixing $2 \mathrm{ml}$ of a phenol red solution $0.5 \%$ (wt/vol) with $16.6 \mathrm{ml}$ of distilled water. The $\mathrm{pH}$ value is then adjusted to 7.8 by adding drops of $1 \mathrm{~N} \mathrm{NaOH}$. A mixture of the phenol red solution and the enzymatic suspension being tested is incubated at $37^{\circ} \mathrm{C}$ for a maximum of 2 hours. After incubation the test results is interpreted as, the color of the wells is turn from red to orange or yellow for all tested strains that is producing carbapenemases, whereas wells corresponding to bacterial extracts of isolates that do not produce carbapenemase remain red. The color will change from red to yellow as early as 5-10 minutes after incubation for KPC producers. In most cases, incubation for 30 minutes is suficient for obtaining a frank color change for carbapenemase producers. The test specificity and sensitivity is $100 \%$ when results are compared with those from molecular-based methods, the reference standard for identifying

1

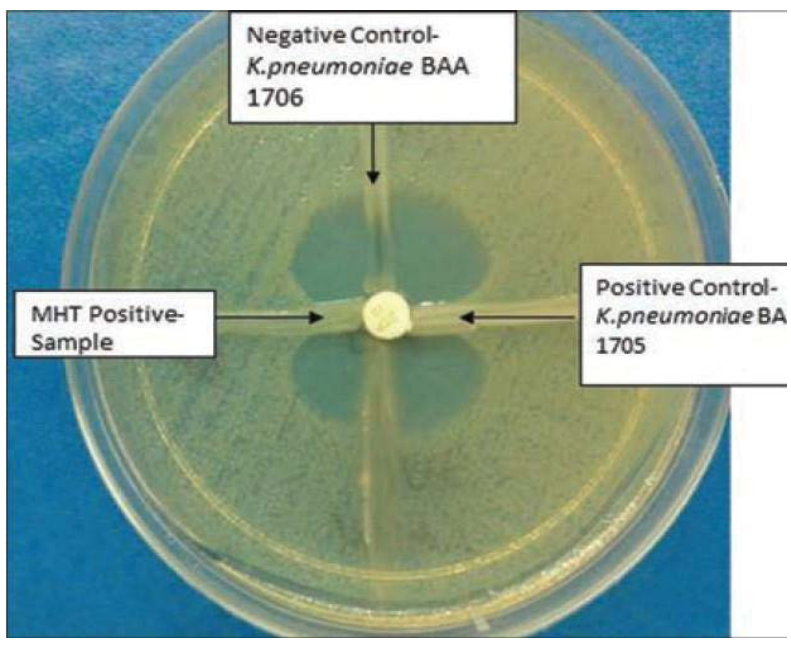

Figure 2: Modified Hodge test. carbapenemase genes [20].

EDTA disk synergy test: EDTA disk synergy test is done with simultaneous testing of $\beta$-lactams (imipenem), for detection of metallo$\beta$-lactamases in the imipenem and meropenem resistant isolates [18]. A $0.5 \mathrm{M}$ EDTA solution is prepared by dissolving $186.1 \mathrm{~g}$ of disodium EDTA in $1000 \mathrm{ml}$ of distilled water. The $\mathrm{pH}$ is adjusted to 8.0 by using $\mathrm{NaOH}$ and is sterilized by autoclaving [21]. An overnight liquid culture of the test isolate is adjusted to a turbidity of $0.5 \mathrm{McFarland}$ standard and spread on the surface of a Mueller Hinton Agar plate. A $10 \mu \mathrm{g}$ imipenen disk (HI - MEDIA) is placed on the agar. A blank disk (6 mm in diameter) is then kept on the inner surface of the lid of the Mueller Hinton Agar plate and $10 \mu \mathrm{l}$ of $0.5 \mathrm{M}$ EDTA is added to it. This EDTA disk is then transferred to the surface of the agar and is kept $10 \mathrm{~mm}$ edge-to-edge apart from the imipenem disk. After incubating overnight at $37^{\circ} \mathrm{C}$, the presence of an expanded growth inhibition zone between the two disks will be interpreted as positive for MBL production. On the same plate about $20 \mathrm{~mm}$ away 2 Imipenem discs is placed about $20 \mathrm{~mm}$ apart. On one of the disc $10 \mu \mathrm{l}$ EDTA solution is added. If the zone enhancement of $5 \mathrm{~mm}$ is observed on the disc with imipenem and EDTA compared to the disc with imipenem alone it implies metallobetalactamase production [21].

2-mercaptopropionic acid inhibition (2-MPA) test: For this test make $0.5 \mathrm{McFarland}$ of standard suspension of test strain in normal saline. After that with the help of sterile swab stick, test strain is streaked as a lawn on the Mueller Hinton Agar plates. Than Two disk of ceftazidime $(30 \mu \mathrm{g})$, two of $10 \mu \mathrm{g}$ imipenem, two of $30 \mu \mathrm{g}$ cefepime are placed at the distance of $50 \mathrm{~mm} .3 \mu \mathrm{l}$ of 2 -mercaptopropionic acid is put on one of the each disk respectively. Plates are incubated at $37^{\circ} \mathrm{C}$ for 24 hrs. The diameter of the growth-inhibitory zone around a beta-lactam disk with 2-meracptopropionic acid is compared with that around the corresponding beta-lactam disk without 2-mercaptopropionic acid. The test is considered positive for the detection of MBL (metallobeta-lactamase) enzyme production when the diameter of the growthinhibitory zone around a beta-lactam disk with 2-mercaptopropionic acid is $\geq 5 \mathrm{~mm}$ larger than that around a disk containing the beta-lactam substrate alone [22].

Boronic acid test for Klebsiella pneumoniae carbapenemases: For the preparation of boronic acid the stock solution is prepared by adding $20 \mu \mathrm{g}$ of phenyl boronic acid in $1 \mathrm{ml}$ of dimethyl sulfoxide. For working solution $20 \mu \mathrm{l}$ of the boronic acid is used (containing $400 \mu \mathrm{g}$ of boronic acid). Two Control strains are used K. pneumonia BAA 1705 (positive control), K. pneumonia BAA 1706 (negative control). Make 0.5 McFarland suspension of test strain in normal saline. With the help of sterile swab stick, test strain is streaked as a lawn on the Mueller Hinton Agar (MHA) plates. The tests is performed by inoculating MHA by the standard diffusion method and placing disks containing eight different beta-lactams (imipenem, meropenem, ertapenem, cefepime, cefoxitin, cefotetan, cefotaxime and ceftazidime) with or without boronic acid onto the agar. The agar plates are incubated at $37^{\circ} \mathrm{C}$ overnight. The diameter of the growth-inhibitory zone around a beta-lactam disk with boronic acid is compared with that around the corresponding betalactam disk without boronic acid. The test is considered positive for the detection of KPC enzyme production when the diameter of the growthinhibitory zone around a beta-lactam disk with boronic acid is $\geq 5 \mathrm{~mm}$ larger than that around a disk containing the beta-lactam substrate alone [23] (Figure 3).

Screening method for carbapenemase production on primary culture plate: At the time of primary culture of specimen on Mac Conkey agar plate a $10 \mu \mathrm{g}$ disc of imipenem is placed on the primary 


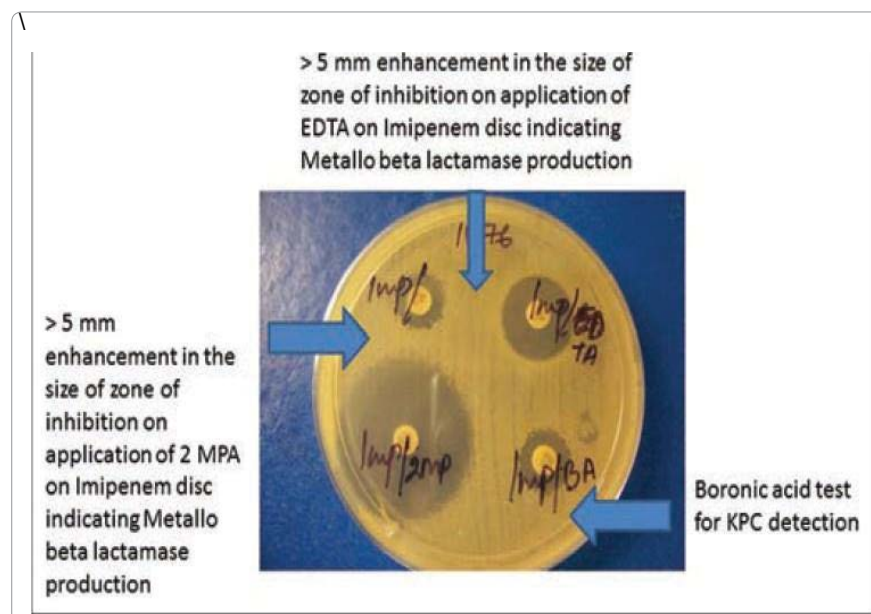

Figure 3: Different methods of detection of metallo-beta-lactamase and Klebsiella pneumonia carbapenemases.

streaks. After 24 hours of incubation when the growth is observed there is no growth within the zone of inhibition if the organism is a non carbapenemase producer. If any colony is observed within this zone of inhibition the clinician can be alerted towards a possible carbapenemase producing isolate from the patient specimen. This is help in more aggressive treatment of the patient especially in ICU as the alert turn-around time is shortened from 48 to 24 hours [24].

UV (Ultra Voilet) spectrophotometer method: Detection of carbapenemase activity can be done using a UV spectrophotometer, which is available in many microbiology laboratories. It is based on several steps, including: (i) an $18 \mathrm{~h}$ culture (which can be shortened in some cases to $8 \mathrm{~h}$ ); (ii) a protein extraction step; and (iii) measurement of imipenem hydrolysis using a UV spectrophotometer [25]. This spectrophotometry-based technique has $100 \%$ sensitivity and 98.5\% specificity for detecting any kind of carbapenemase activity [25]. This cheap technique can accurately differentiate carbapenemase producers from non-carbapenemase producers among carbapenemnon-susceptible isolates [outer membrane permeability defect, overproduction of cephalosporinases or/and extended-spectrum $\beta$-lactamases (ESBLs)]. It can be implemented in any reference laboratory, but this technique still requires time. Recently, the use of mass spectrometry for detection of carbapenemase activity has been proposed, based on the analysis of the degradation of a carbapenem molecule [26]. Although this technique has to be further evaluated, matrix-assisted laser desorption ionization time-of-flight mass spectrometry (MALDI-TOF) equipment is increasingly used in the diagnostic bacteriology laboratory.

Molecular testsfor carbapenemase genesidentification: Molecular techniques remain the gold standard for the precise identification of carbapenemase genes [27]. Most of these techniques are based on PCR and may be followed by a sequencing step if a precise identification of the carbapenemase gene is needed (e.g. VIM type, KPC type, NDM type or OXA-48 type) [28]. They are either single or multiplex PCR techniques. A PCR technique performed directly on colonies can give results within 4-6 hrs (or less when using real-time PCR technology) with excellent sensitivity and specificity. Similarly, other molecular techniques are useful for this purpose [29]. The main disadvantages of the molecular-based technologies are their cost, the requirement for trained microbiologists and the inability to detect novel unidentified genes. Sequencing of the genes is interesting mostly for research and epidemiological purposes.

\section{Conclusion}

Carbapenem resistance in Gram-negative organisms is increasingly encountered in healthcare-associated infections in India. Facing the global crisis in antibiotic resistance, presented by rapid dissemination of carbapenemase producing Gram-negative bacteria, many issues remain controversial, especially detection methods and treatment options. However, active surveillance, hand hygiene, contact precautions, and appropriate antibiotic usage are part of effective approach in reducing incidence of colonization and infections caused by these life treating microorganisms. Nonmolecular tests for detection of carbapenemases have variable results for Modified Hodge Test, EDTA disk synergy test, MIC by Agar Dilution Test and MIC by E-test. Out of these four tests the Modified Hodge Test often lacks specificity (false positive results for high-level AmpC producers) and sensitivity (weak detection of NDM producers). But this test works well for detection of KPC and OXA-48 producers. The spectrophotometry-based technique has $100 \%$ sensitivity and $98.5 \%$ specificity for detecting any kind of carbapenemase activity. This cheap technique can accurately differentiate carbapenemase producers from non-carbapenemase producers. Although PCR-based methods have been proven to be highly sensitive and reliable for rapid diagnosis of carbapenemase producers but these methods are costly and require expertise that is not readily available in many centers.

\section{References}

1. Queenan AM, Bush K (2007) Carbapenemases: the versatile beta-lactamases Clin Microbiol Rev 20: 440-458.

2. Rice LB, Bonomo RA (2005) Genetic and biochemical mechanisms of bacterial resistance to antimicrobial agents. In: Lorian $\mathrm{V}$, ed. Antibiotics in laboratory medicine. Philadelphia: Lippincott Williams \& Wilkins. 5: 441-508.

3. Thomson KS (2010) Extended-spectrum-beta-lactamase, AmpC, and Carbapenemase issues. J Clin Microbiol 48: 1019-1025.

4. Pitout JD, Laupland KB (2008) Extended-spectrum beta-lactamase-producing Enterobacteriaceae: an emerging public-health concern. Lancet Infect Dis 8 159-166.

5. Lartigue MF, Poirel L, Poyart C, Réglier-Poupet H, Nordmann P (2007) Ertapenem resistance of Escherichia coli. Emerg Infect Dis 13: 315-317.

6. Donskey CJ (2006) Antibiotic regimens and intestinal colonization with antibiotic-resistant gram-negative bacilli. Clin Infect Dis 43: S62-69.

7. Miriagou V, Cornaglia G, Edelstein M, Galani I, Giske CG, et al. (2010) Acquired carbapenemases in Gram-negative bacterial pathogens: detection and surveillance issues. Clin Microbiol Infect 16: 112-122.

8. Centers for Disease Control and Prevention (CDC) (2009) Guidance for control of infections with carbapenem-resistant or carbapenemase-producing Enterobacteriaceae in acute care facilities. MMWR Morb Mortal Wkly Rep 58: 256-260.

9. Paterson DL (2006) Resistance in gram-negative bacteria: Enterobacteriaceae Am J Infect Control 34: S20-28.

10. Gupta N, Limbago BM, Patel JB, Kallen AJ (2011) Carbapenem-resistant Enterobacteriaceae: epidemiology and prevention. Clin Infect Dis 53: 60-67.

11. Pitout JD, Laupland KB (2008) Extended-spectrum beta-lactamase-producing Enterobacteriaceae: an emerging public-health concern. Lancet Infect Dis 8 : 159-166.

12. Paterson DL (2006) Resistance in gram-negative bacteria: Enterobacteriaceae Am J Infect Control 34: S20-28

13. Nordmann P, Dortet L, Poirel L (2012) Carbapenem resistance in Enterobacteriaceae: here is the storm! Trends Mol Med 18: 263-272.

14. Andrews JM (2001) Determination of minimum inhibitory concentrations. J Antimicrob Chemother 48 Suppl 1: 5-16.

15. Joyce LF, Downes J, Stockman K, Andrew JH (1992) Comparison of five 
Citation: Kaur M, Gupte S, Kaur T (2015) Clinical Importance of Carbapenemase Production in Gram-Negative Bacteria. J Trop Dis 3: 164. doi:10.4172/2329891X.1000164

Page 5 of 5

methods including the PDM Epsilometer test ( $E$ test), for antimicrobial susceptibility testing of Pseudomonas aeruginosa. J Clin Microbiol 30: 27092713.

16. Turnidge JD, Ferraro MJ, Jorgensen JH (2003) Susceptibility Test Methods: General Considerationa. In PR Murray, EJ Baron, JH Jorensen, MA Pfaller $\mathrm{RH}$ Yolken (eds) Manual of Clinical Microbiology, American Society of Clinical Microbiology, Washington, USA.

17. Davison HC, Low JC, Woolhouse ME (2000) What is antibiotic resistance and how can we measure it? Trends Microbiol 8: 554-559.

18. Lee K, Chong Y, Shin HB, Kim YA, Yong D, et al. (2001) Modified Hodge and EDTA -disk synergy tests to screen metallo-lactamase-producing strains of Pseudomonas and Acinetobacter species. Clin Microbiol Infect 7: 88-91.

19. Clinical and Laboratory Standards Institute (2009) Performance standards for antimicrobial disk susceptibility tests; Approved standard (10th edn), Clinical and Laboratory standard Institute, Wayne, Pennsylvania, USA

20. Nordmann P, Poirel L, Dortet L (2012) Rapid detection of carbapenemaseproducing Enterobacteriaceae. Emerg Infect Dis 18: 1503-1507.

21. Yong D, Lee K, Yum JH, Shin HB, Rossolini GM, et al. (2002) Imipenem-EDTA disk method for differentiation of metallo-beta-lactamase-producing clinical isolates of Pseudomonas spp. and Acinetobacter spp. J Clin Microbiol 40: 3798-3801.

22. Thomson KS (2010) Extended-spectrum-beta-lactamase, AmpC, and Carbapenemase issues. J Clin Microbiol 48: 1019-1025.
23. Arnold RS, Thom KA, Sharma S, Phillips M, Kristie Johnson J, et al. (2011) Emergence of Klebsiella pneumoniae carbapenemase-producing bacteria South Med J 104: 40-45.

24. Amos Adler, Shiri Navon-Venezia, Jacob Moran-Gilad, Evgeniya Marcos, David Schwartz, et al. (2011) Laboratory and Clinical Evaluation of Screening Agar Plates for Detection of Carbapenem-Resistant Enterobacteriaceae from Surveillance Rectal Swabs. J of Clin Microbiol. 49: 2239-2242.

25. Bernabeu S, Poirel L, Nordmann P (2012) Spectrophotometry-based detection of carbapenemase producers among Enterobacteriaceae. Diagn Microbiol Infect Dis 74: 88-90.

26. Burckhardt I, Zimmermann S (2011) Using matrix-assisted laser desorption ionization-time of flight mass spectrometry to detect carbapenem resistance within 1 to 2.5 hours. J Clin Microbiol. 49: 3321-3324.

27. Nordmann P, Gniadkowski M, Giske CG, Poirel L, Woodford N, et al. (2012) Identification and screening of carbapenemase-producing Enterobacteriaceae. Clin Microbiol Infect 18: 432-438.

28. Poirel L, Walsh TR, Cuvillier V, Nordmann P (2011) Multiplex PCR for detection of acquired carbapenemase genes. Diagn Microbiol Infect Dis 70: 119-123.

29. Cuzon G, Naas T, Bogaerts P, Glupczynski Y, Nordmann P (2012) Evaluation of a DNA microarray for the rapid detection of extended-spectrum $\beta$-lactamases (TEM, SHV and CTX-M), plasmid-mediated cephalosporinases (CMY-2-like, DHA, FOX, ACC-1, ACT/MIR and CMY-1-like/MOX) and carbapenemases (KPC, OXA-48, VIM, IMP and NDM). J Antimicrob Chemother 67: 1865-1869. 